\title{
Endoscopic Ultrasound-Guided Treatment of Pancreatic Cystic and Solid Masses
}

\author{
Jaihwan Kim \\ Department of Internal Medicine, Seoul National University Bundang Hospital, Seoul National University College of Medicine, Seongnam,
} Korea

Pancreatic tumor is one of the most difficult diseases to diagnose and treat because of its anatomical location and characteristics. Recently, there have been several innovative trials on the treatment of pancreatic tumors using endoscopic ultrasound (EUS) because it allows selective access to the difficult to reach target organ along the gastrointestinal tract and can differentiate vessels by color Doppler. Among these trials, several have investigated EUS-guided ethanol lavage with or without paclitaxel for pancreatic cystic tumors. These studies show a 33\% to $79 \%$ complete resolution rate with a favorable safety profile. Compared to EUS-guided ethanol lavage for pancreatic cystic tumors, EUS-guided radiofrequency ablation is considered a less invasive treatment method for pancreatic cancer. Although there are still several difficulties and concerns about complications, one clinical study reported $72.8 \%$ feasibility with favorable safety, and therefore, we anticipate the results of ongoing studies with these new less invasive techniques. Clin Endosc 2015;48:308-311

Key Words: Endosonography; Pancreatic cyst; Pancreatic neoplasms; Ablation

\section{INTRODUCTION}

Pancreatic tumor is one of the most difficult diseases to diagnose and treat because of its anatomical location and characteristics. However, the recent advent of endoscopic ultrasound (EUS) and EUS-guided fine needle aspiration has advanced the diagnosis of pancreatic tumors. Moreover, there is an effort to use EUS not only as a diagnostic modality but also as a therapeutic intervention for procedures such as pseudocyst drainage or celiac nerve plexus block.

Recently, there have been several innovative trials on the treatment of pancreatic tumors using EUS because it allows selective access to the difficult to reach target organ along the gastrointestinal tract and can differentiate vessels by color

Received: April 17, 2015 Revised: June 20, 2015

Accepted: June 22, 2015

Correspondence: Jaihwan Kim

Department of Internal Medicine, Seoul National University Bundang Hospital, Seoul National University College of Medicine, 82 Gumi-ro 173beon-gil, Bundang-gu, Seongnam 463-707, Korea

Tel: +82-31-787-7075, Fax: +82-31-787-4290, E-mail: drjaihwan@gmail.com

(c) This is an Open Access article distributed under the terms of the Creative Commons Attribution Non-Commercial License (http://creativecommons.org/ licenses/by-nc/3.0) which permits unrestricted non-commercial use, distribution, and reproduction in any medium, provided the original work is properly cited.
Doppler. Investigated EUS-guided oncologic interventions are as follows: EUS-guided ethanol lavage (EUS-EL), EUS-guided radiofrequency ablation (EUS-RFA), EUS-guided photodynamic therapy, EUS-guided Nd:YAG laser, EUS-guided high intensity focused ultrasound, EUS-guided brachytherapy, EUS-guided fiducial marker insertion, and EUS-guided antitumor agent injection. ${ }^{1}$ Among these, EUS-EL and EUS-RFA will be discussed in this article.

\section{EUS-EL}

The prevalence of pancreatic cysts is $2.5 \%$ in the general population and $8 \%$ to $10 \%$ in elderly people. ${ }^{2,3}$ Among various pancreatic cysts, differentiation between mucinous and non-mucinous cysts is important because of the malignant potential of mucinous cystic tumor. However, it is not easy to differentiate them. One study reported that up to $50 \%$ of cysts are indeterminate despite a variety of radiologic examinations with cystic fluid analysis. ${ }^{4}$

International guidelines have recently been published for the management of mucinous cystic neoplasm, a tumor with malignant potential. ${ }^{5}$ The guidelines detail the method and 
timing of surgery; however, they do not suggest how patients who are not suitable for surgery should be managed. Therefore, there was a necessity for minimally invasive therapy.

Percutaneous ethanol lavage has been used in the liver, ${ }^{6}$ kidney, and thyroid gland ${ }^{8}$ for a long time and is established as a safe and effective treatment. Ethanol is inexpensive, widely available, and little viscous. Theoretical mechanisms of ethanol lavage are lysis of the cyst membrane, protein denaturation, and vascular occlusion. Based on experience with percutaneous ethanol injection, pancreatic tissue ablation by EUS-EL was studied. The technique used was as follows: the cyst was punctured via a transduodenal or transgastric route and cystic fluid aspiration was performed using a curvilinear array echoendoscope and 22-gauge needle. After subtotal evacuation of cystic fluid, injection of ethanol, which equals to that of aspiration, was performed (Fig. 1). Then, lavage of the cyst was performed for 3 to 5 minutes. In some studies, an additional paclitaxel injection was administered after the final ethanol lavage; paclitaxel is a well-known chemotherapeutic agent that inhibits microtubule dependent cell division and because of its hydrophobic and viscous nature, it was expected to be retained in the

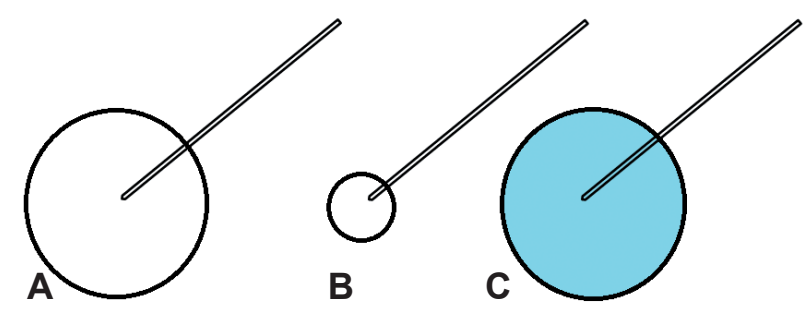

Fig. 1. Endoscopic ultrasound-guided ethanol lavage. (A) The cyst is punctured by a fine needle. (B) Subtotal evacuation of the cystic fluid is performed. (C) Injection of ethanol (blue), which equals to that of aspiration, is performed. cyst without leakage.

The first study of EUS-EL, by Gan et al., ${ }^{9}$ reported safety and feasibility of EUS-EL. In this study, $5 \%$ to $80 \%$ ethanol was used and $35 \%$ of patients had complete resolution. The authors concluded that ethanol lavage of pancreatic cystic lesions is safe and feasible. The next study, by Oh et al., ${ }^{10}$ evaluated the safety, feasibility, and response after EUSEL with additional paclitaxel injection. In this study, they reported $79 \%$ complete resolution. The next study, by Oh et al., ${ }^{11}$ investigated the effectiveness of ethanol and paclitaxel in septated cysts, which were considered a negative influencing factor of EUS-EL. In this study, the authors reported $60 \%$ complete resolution and concluded that EUSEL was effective in septated cysts. In the same year, DeWitt et al. ${ }^{12}$ published a randomized, double-blind study which compared ethanol versus saline. The authors concluded that EUS-EL resulted in a greater decrease in pancreatic cyst size compared with saline solution lavage with a similar safety profile. Complete pancreatic cyst ablation was observed in $33.3 \%$ of patients. In 2011, Oh et al. ${ }^{4}$ reported the long-term outcomes of EUS-EL with paclitaxel. They reported that $62 \%$ of patients had complete resolution with a median 21.7 months of follow-up and determined that small cyst volume was predictive of complete resolution. ${ }^{4}$ In the same year, DiMaio et al. ${ }^{13}$ reported that two EUS-EL procedures resulted in a significantly greater decrease in the size and surface area of pancreatic cysts and was associated with a significantly higher rate of image-defined cyst resolution than only one procedure. In 2014, DeWitt et al. ${ }^{14}$ reported that EUS-EL with paclitaxel may possibly eliminate mutant DNA in neoplastic pancreatic cysts and demonstrated that it led to complete resolution in $50 \%$ of cysts. In addition, this was the first study to use additional paclitaxel in a Western country. The

Table 1. Summary of Studies of Endoscopic Ultrasound-Guided Ethanol Ablation with or without Paclitaxel

\begin{tabular}{|c|c|c|c|c|c|}
\hline Study & $\begin{array}{l}\text { No. of } \\
\text { Patients }\end{array}$ & Ablative agent & $\begin{array}{c}\text { FU period, median } \\
\text { (range) }\end{array}$ & Resolution rate & Etc. \\
\hline Gan et al. ${ }^{9}(2005)$ & 25 & $5 \%-80 \% \mathrm{EtOH}$ & $6-12 \mathrm{mo}$ & $\begin{array}{c}35 \% \text { CR }(8 / 23) \\
7 \% \text { PR }(2 / 23)\end{array}$ & 1st study \\
\hline Oh et al. ${ }^{10}(2008)$ & 14 & $\begin{array}{c}80 \% / 90 \% \mathrm{EtOH}+ \\
\text { paclitaxel }\end{array}$ & 9 mo $(6-23)$ & $\begin{array}{c}79 \% \text { CR }(11 / 14) \\
14 \% \text { PR }(2 / 14)\end{array}$ & $\mathrm{EtOH}+$ paclitaxel \\
\hline Oh et al. ${ }^{11}(2009)$ & 10 & 99\% EtOH+paclitaxel & $8.5 \mathrm{mo}(6-18)$ & $\begin{array}{l}60 \% \text { CR }(6 / 10) \\
20 \% \operatorname{PR}(2 / 10)\end{array}$ & Septated cysts \\
\hline DeWitt et al. ${ }^{12}(2009)$ & 42 & $80 \% \mathrm{EtOH}$ & 3-4 mo after 2nd lavage & $33 \%$ CR (12/36) & EtOH vs. saline \\
\hline Oh et al. ${ }^{4}(2011)$ & 47 & 99\% EtOH+paclitaxel & 20 mo (12-44) & $\begin{array}{c}62 \% \text { CR }(29 / 47) \\
13 \% \text { PR }(6 / 47)\end{array}$ & $\begin{array}{l}\text { Long term outcome } \\
\text { \& response predictor }\end{array}$ \\
\hline DiMaio et al. $^{13}(2011)$ & 13 & $80 \% \mathrm{EtOH}$ & 13 mo after 1st lavage & $38 \%$ CR (5/13) & Multiple ablations \\
\hline DeWitt et al. ${ }^{14}(2014)$ & 22 & $100 \% \mathrm{EtOH}+$ paclitaxel & 27 mo (17-42) & $\begin{array}{c}50 \% \text { CR }(10 / 20) \\
25 \% \text { PR }(5 / 20)\end{array}$ & Cyst DNA analysis \\
\hline
\end{tabular}

FU, follow-up; EtOH, ethanol; CR, complete response; PR, partial response. 
results of EUS-EL studies are summarized in Table 1.

Most complications observed were mild and manageable with conservative treatment. Common complications were mild abdominal pain (10\%), intracystic bleeding (1\%), pericystic spillage $(2 \%)$, and pancreatitis $(3 \%) .{ }^{15}$ However, there was one case of portal vein thrombosis after EUS-EL with paclitaxel which required pylorus-preserving pancreaticoduodenectomy and portal vein thrombectomy ${ }^{15}$ and therefore, the possibility of rare and severe complications should be kept in mind. In addition, the safety of paclitaxel was an important concern in studies where paclitaxel was administered; Oh et al. ${ }^{16}$ reported that plasma paclitaxel concentration after EUSEL with paclitaxel was nearly as low as the undetectable value and concluded that paclitaxel was expected to rarely cause systemic side-effects.

Despite the acceptable therapeutic effect and safety of EUSEL with or without paclitaxel observed in these studies, there are still several debates. ${ }^{17}$ First, there is controversy regarding observation versus EUS-EL because it is impossible to definitively diagnose pancreatic cyst without surgery and as such, some critics state that there is a possibility of unnecessary treatment. The second concern of critics is whether complete remission on imaging work up such as computerized tomography guarantees pathological complete elimination because it is very difficult to evaluate the treated lesion. In addition, there is a possibility of malignancy not from the treated lesion but from another part of pancreas. The last concern is the possibility of incomplete ablation of malignant or premalignant lesions because studies have reported variable histopathological responses from $0 \%$ to $100 \%$ in cases of surgery after EUS-EL. ${ }^{4}$

\section{EUS-RFA}

In spite of surgical and oncological advances in the treatment of pancreatic cancer, the prognosis of the disease is stagnated and grave. Although there are several reasons for this, one reason is that only $10 \%$ of patients have a chance to receive a curative intent surgery. A systemic review reported that planned palliative $\mathrm{R} 2$ resections in patients with pancreatic cancer resulted in increased morbidity, mortality, and longer hospital and therefore concluded that the procedure was not justified; the pooled median survival times were 8.2 months for R2 resection and 6.7 months for palliative bypass procedures but the difference was not statistically significant. $^{18}$ Therefore, there is a need for less invasive therapy instead of palliative resection.

RFA is a well-established anti-tumor treatment using local thermal-induced coagulative necrosis. It is recognized as one of the least invasive therapeutic options for pancreatic cancer. ${ }^{1,19,20}$ However, it should be considered that the pancreas is different from other organs, especially the liver. The first difference is that the physical properties of the pancreas are different from those of the liver and therefore, the RFA protocol for the liver cannot be used in the pancreas. There have been many ex vivo and in vivo studies to determine the optimal thermal kinetic characteristics; however, there is still no standardized protocol for pancreatic RFA. ${ }^{20}$ The second difference is in its location. The pancreas, especially the head, is surrounded by the stomach, duodenum, portal vein, superior mesenteric vessels, and bile duct, and as such pancreatic RFA has a risk of thermal injury to surrounding organs. The last difference is in tumor biology. Pancreatic cancer usually has diffuse margins compared to hepatocellular carcinoma or hepatic metastasis, which have discrete margins. As a result, it is difficult to ablate pancreatic cancer completely and two or more procedures may be necessary in many cases.

For pancreatic RFA, there have been several intraoperative RFA studies and one recent EUS-RFA study. ${ }^{21}$ Therefore, the outcomes are mainly from intraoperative RFA. Although all studies have demonstrated that RFA leads to tumor necrosis and a decrease in tumor volume, it is difficult to summarize because of the diversity among the studies. ${ }^{19,21}$ Early clinical studies were associated with unacceptably high rates of morbidity ( $0 \%$ to $40 \%)$ and mortality ( $0 \%$ to $25 \%)$. However, complications were markedly reduced in more recent studies because of the use of a cooling system and surgical diversion. Compared to studies on intraoperative RFA, most studies of EUS-RFA are animal studies for feasibility, ${ }^{22-24}$ safety, and optimal settings (Fig. 2). In these studies, the authors concluded that EUS-RFA was feasible and safe in vivo. Recently, one study reported the use of EUS-RFA with the cryotherm probe (ERBE Elektromedizin GmbH, Tübingen, Germany) in 22 patients with locally advanced pancreatic cancer; ${ }^{25}$ the authors concluded that EUS-RFA was successful in 16 patients $(72.8 \%)$ and that complications were manageable.

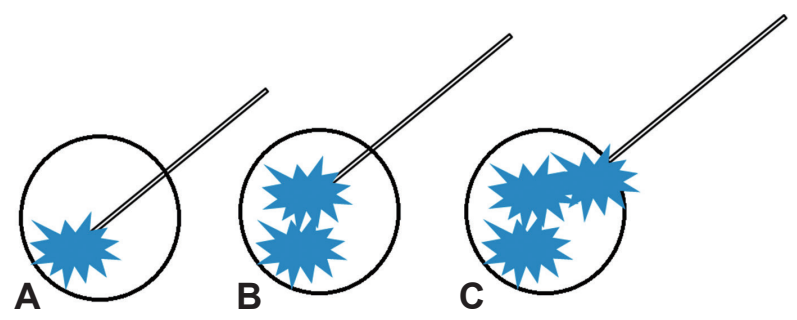

Fig. 2. Endoscopic ultrasound-guided radiofrequency ablation. (A) The distal part of the mass is ablated by a radiofrequency ablating needle. (B) The more proximal part of the mass is ablated. (C) According to needle withdrawal, ablation is repetitively performed in the proximal part of the mass. 


\section{CONCLUSIONS}

EUS-EL for pancreatic cysts has demonstrated acceptable outcomes with favorable safety. However, the results are still limited because of small sample sizes and relatively short-term data. There is a necessity for large prospective scale studies. Meanwhile, EUS-RFA is a currently evolving technique that is expected to be complimentary to the current standard therapy of pancreatic cancer; however, more data and studies are still necessary. In addition to pancreatic cyst or cancer treatment, we also anticipate the results of ongoing studies targeting benign solid tumors such as neuroendocrine tumors or solid pseudopapillary tumors with these less invasive techniques.

\section{Conflicts of Interest}

The author has no financial conflicts of interest.

\section{REFERENCES}

1. Suzuki R, Irisawa A, Bhutani MS. Endoscopic ultrasound-guided oncologic therapy for pancreatic cancer. Diagn Ther Endosc 2013;2013:157581.

2. Laffan TA, Horton KM, Klein AP, et al. Prevalence of unsuspected pancreatic cysts on MDCT. AJR Am J Roentgenol 2008;191:802-807.

3. de Jong K, Nio CY, Hermans JJ, et al. High prevalence of pancreatic cysts detected by screening magnetic resonance imaging examinations. Clin Gastroenterol Hepatol 2010;8:806-811.

4. Oh HC, Seo DW, Song TJ, et al. Endoscopic ultrasonography-guided ethanol lavage with paclitaxel injection treats patients with pancreatic cysts. Gastroenterology 2011;140:172-179.

5. Tanaka M, Fernández-del Castillo C, Adsay V, et al. International consensus guidelines 2012 for the management of IPMN and MCN of the pancreas. Pancreatology 2012;12:183-197.

6. Larssen TB, Viste A, Jensen DK, Søndenaa K, Røkke O, Horn A. Single-session alcohol sclerotherapy in benign symptomatic hepatic cysts. Acta Radiol 1997;38:993-997.

7. Bean WJ. Renal cysts: treatment with alcohol. Radiology 1981;138:329331.

8. Bennedbaek FN, Hegedüs L. Treatment of recurrent thyroid cysts with ethanol: a randomized double-blind controlled trial. J Clin Endocrinol Metab 2003;88:5773-5777.

9. Gan SI, Thompson CC, Lauwers GY, Bounds BC, Brugge WR. Ethanol lavage of pancreatic cystic lesions: initial pilot study. Gastrointest Endosc 2005;61:746-752.

10. Oh HC, Seo DW, Lee TY, et al. New treatment for cystic tumors of the pancreas: EUS-guided ethanol lavage with paclitaxel injection. Gastrointest Endosc 2008;67:636-642.

11. Oh HC, Seo DW, Kim SC, et al. Septated cystic tumors of the pancreas: is it possible to treat them by endoscopic ultrasonography-guided intervention? Scand J Gastroenterol 2009;44:242-247.

12. DeWitt J, McGreevy K, Schmidt CM, Brugge WR. EUS-guided ethanol versus saline solution lavage for pancreatic cysts: a randomized, double-blind study. Gastrointest Endosc 2009;70:710-723.

13. DiMaio CJ, DeWitt JM, Brugge WR. Ablation of pancreatic cystic lesions: the use of multiple endoscopic ultrasound-guided ethanol lavage sessions. Pancreas 2011;40:664-668.

14. DeWitt JM, Al-Haddad M, Sherman S, et al. Alterations in cyst fluid genetics following endoscopic ultrasound-guided pancreatic cyst ablation with ethanol and paclitaxel. Endoscopy 2014;46:457-464.

15. Oh HC, Seo DW, Kim SC. Portal vein thrombosis after EUS-guided pancreatic cyst ablation. Dig Dis Sci 2012;57:1965-1967.

16. Oh HC, Seo DW, Kim SH, Min B, Kim J. Systemic effect of endoscopic ultrasonography-guided pancreatic cyst ablation with ethanol and paclitaxel. Dig Dis Sci 2014;59:1573-1577.

17. Oh HC, Brugge WR. EUS-guided pancreatic cyst ablation: a critical review (with video). Gastrointest Endosc 2013;77:526-533.

18. Gillen S, Schuster T, Friess H, Kleeff J. Palliative resections versus palliative bypass procedures in pancreatic cancer: a systematic review. Am J Surg 2012;203:496-502.

19. Fabbri C, Luigiano C, Lisotti A, et al. Endoscopic ultrasound-guided treatments: are we getting evidence based: a systematic review. World J Gastroenterol 2014;20:8424-8448.

20. Pandya GJ, Shelat VG. Radiofrequency ablation of pancreatic ductal adenocarcinoma: the past, the present and the future. World J Gastrointest Oncol 2015;7:6-11.

21. Keane MG, Bramis K, Pereira SP, Fusai GK. Systematic review of novel ablative methods in locally advanced pancreatic cancer. World J Gastroenterol 2014;20:2267-2278.

22. Goldberg SN, Mallery S, Gazelle GS, Brugge WR. EUS-guided radiofrequency ablation in the pancreas: results in a porcine model. Gastrointest Endosc 1999;50:392-401.

23. Kim HJ, Seo DW, Hassanuddin A, et al. EUS-guided radiofrequency ablation of the porcine pancreas. Gastrointest Endosc 2012;76:1039-1043.

24. Gaidhane M, Smith I, Ellen K, et al. Endoscopic ultrasound-guided radiofrequency ablation (EUS-RFA) of the pancreas in a porcine model. Gastroenterol Res Pract 2012;2012:431451.

25. Arcidiacono PG, Carrara S, Reni M, et al. Feasibility and safety of EUS-guided cryothermal ablation in patients with locally advanced pancreatic cancer. Gastrointest Endosc 2012;76:1142-1151. 